02,05, 12

\title{
Сверхпроводящие свойства In, наноструктурированного в порах тонких пленок из микросфер $\mathrm{SiO}_{2}$
}

\author{
(ㄱ Н.Ю. Михайлин, С.Г. Романов, Ю.А. Кумзеров, А.В. Фокин, Д.В. Шамшур \\ Физико-технический институт им. А.Ф. Иофрфе РАН, \\ Санкт-Петербург, Россия, \\ E-mail: mikhailin.nik@gmail.com
}

(Поступила в Редакцию 16 апреля 2018 г.)

\begin{abstract}
Впервые получены образцы сверхпроводящего индиевого нанокомпозита на основе тонкопленочной пористой диэлектрической матрицы, приготовленной методом Лэнгмюра-Блоджетт, изучены их низкотемпературные электрофизические и магнитные свойства. Пленки толщиной $b \leq 5 \mu \mathrm{m}$ изготавливались из сфер оксида кремния диаметром $D=200 \mathrm{~nm}$ и $250 \mathrm{~nm}$, индий вводился в поры пленок из расплава под давлением $P \leq 5 \mathrm{kbar}$. Таким образом в порах создавалась трехмерная слабоупорядоченная структура наногранул индия, образующая сплошную токопроводящую сеть. Измерения температурных и магнитополевых зависимостей сопротивления и магнитного момента образцов показали рост критических параметров сверхпроводящего состояния наноструктурированного индия (критическая температура $T_{c} \leq 3.62 \mathrm{~K}$ и критическое магнитное поле $H_{\mathrm{c}}$ при $\left.T=0 \mathrm{~K} H_{\mathrm{c}}(0) \leq 1700 \mathrm{Oe}\right)$ относительно массивного материала $\left(T_{\mathrm{c}}=3.41 \mathrm{~K}, H_{c}(0)=280 \mathrm{Oe}\right)$. В зависимостях сопротивления от температуры и магнитного поля наблюдался ступенчатый переход в сверхпроводящее состояние, связанный со структурой нанокомпозита. В зависимости магнитного момента $M$

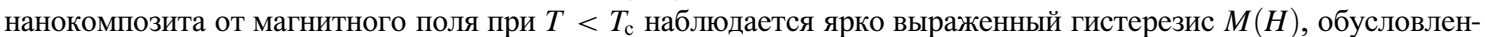
ный многосвязной структурой токопроводящей индиевой сетки. Полученные результаты интерпретированы с учетом размерной зависимости сверхпроводящих характеристик нанокомпозита.
\end{abstract}

Работа частично выполнена при поддержке Программы Президиума РАН № 1.4 „Актуальные проблемы физики низких температур“.

DOI: 10.21883/FTT.2018.10.46515.109

\section{1. Введение}

Общей задачей исследований являлось получение сверхпроводящих (СП) нанокомпозитов на основе пористых матриц и сравнительное изучение особенностей их СП-свойств в материалах с различными характерными диаметрами токопроводящей сетки и степенью упорядоченности решетки. В данной работе были изучены сверхпроводящие свойства индиевой сетки, образованной в порах опалоподобных пленок, полученных методом Лэнгмюра-Блоджетт. Ранее были исследованы сверхпроводящие (СП) нанокомпозиты индий-опал с высокой степенью регулярности (рис. 1, левая часть), которые демонстрируют увеличение температуры сверхпроводящего перехода $T_{\mathrm{c}}$ вплоть до $T_{\mathrm{c}} \approx 4.5 \mathrm{~K}$ и позволяют получить СП-материал, критическое магнитное поле достигает величины $H_{\mathrm{c}}(T=0 \mathrm{~K}) \leq 22 \mathrm{kOe}$ [1]. Отметим, что в чистом массивном индии параметры СП состояния составляют $T_{\mathrm{c}}=3.41 \mathrm{~K}$ и $H_{c}(T=0 \mathrm{~K})=280 \mathrm{Oe}$. Результат достигался путем организации в пространстве массивной диэлектрической матрицы опала регулярного трехмерного ансамбля наногранул индия, соединенных тонкими мостиками нанометрового $(d \approx 10-45 \mathrm{~nm})$ диаметра.

В настоящей работе мы изучали температурные и магнитополевые зависимости электросопротивления $R(T, H)$ и магнитного момента $M(T, H)$ сверхпроводящего нанокомпозита, сформированного из расплава индия под давлением в полостях тонкопленочной диэлектрической матрицы. Матрица представляла собой тонкие слои различной толщины $b \approx 1.3$ и $5 \mu \mathrm{m}$, сформированные из сфер $\mathrm{SiO}_{2}$ методом принудительной кристаллизации [2] (рис. 1, правая часть). Получившаяся в результате металлическая реплика при общем сходстве с репликой опала обладает, в отличие от него, набором характерных диаметров, что следует рассматривать как нарушение регулярности. Нашей задачей было исследование влияния наноструктурирования на СП характеристики полученных образцов. В целом данный подход можно определить как топологическое конструирование сверхпроводящих материалов, ставящее фундаментальные параметры сверхпроводника в зависимость от структуры нанокомпозита.

\section{2. Экспериментальные результаты и их обсуждение}

Нами был исследован CП In-нанокомпозит, приготовленный на основе матрицы, полученной путем принудительной кристаллизации - методом ЛэнгмюраБлоджетт (ЛБ) [2] (рис. 1, правая часть). Кристаллизация была достигнута путем формирования монослоя гексагонально упакованных силикатных сфер на поверхности воды и последующего переноса этого монослоя на подложку. Затем процесс повторяли несколько раз 


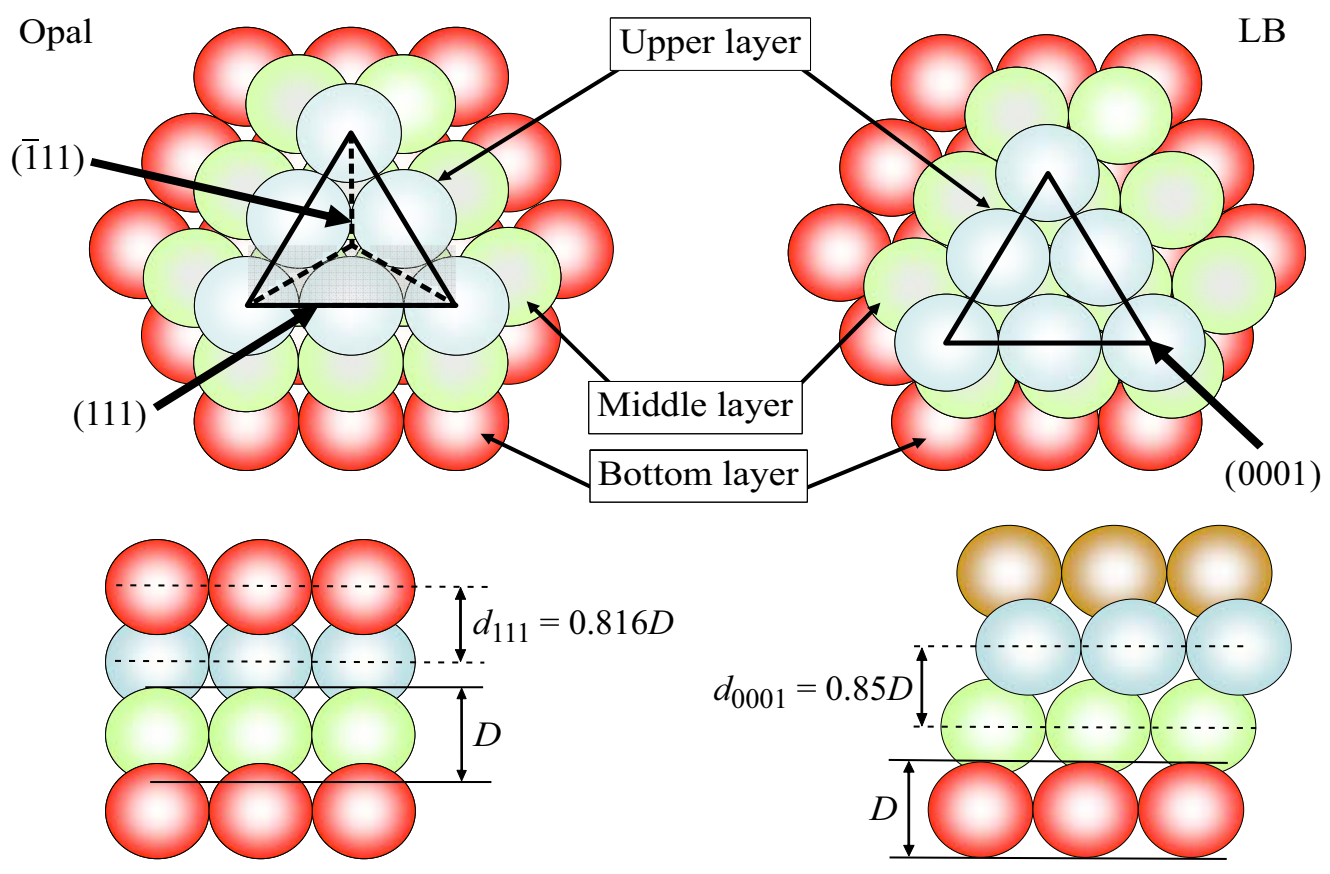

Рис. 1. Схематическое изображение структуры опала (слева) и матрицы, изготовленной методом Лэнгмюра-Блоджетт (правая часть).

для получения слоисто-трехмерной системы. Далее, в полученный ЛБ кристалл, как в матрицу, вводили индий из расплава в условиях гидростатического давления до $5 \mathrm{kbar}$ (аналогично [3]).

Матрица ЛБ (рис. 1, правая часть), заполненная индием, состоит из гранул сверхпроводника, размер которых ограничен сверху характерным размером сферы. Крупные гранулы соединены относительно тонкими мостиками (перетяжками), поперечные и продольные размеры которых варьируются в широких пределах из-за продольного смещения монослоев сфер в ЛБ-матрице. Таким образом, перетяжки могут иметь диаметр от 10 до $100 \mathrm{~nm}$. Реализуется перколяционная модель проводящей решетки, отличие которой от классической состоит в жестком ограничении сверху как размеров гранул, так и размеров мостиков.

Нами были изготовлены СП-пленочные In-ЛБ нанокомпозиты двух типов. На стеклянных подложках методом ЛБ были сформированы слои шаров $\mathrm{SiO}_{2}$ из 5 слоев (ЛБ5, диаметр шаров $D=250 \mathrm{~nm}$ ) и 27 слоев (ЛБ27, диаметр шаров $D=200 \mathrm{~nm}$ ). Для получения СП нанокомпозита пустоты слоев были заполнены индием из расплава [3]. Заполнение проводилось с торцов образцов, чтобы исключить поверхностное покрытие пленки индием.

На рис. 2 представлены микрофотографии участков поверхности нанокомпозитов, полученных с использованием сканирующего электронного микроскопа (SEM). Хорошо видна относительная регулярность структуры матрицы (более ярко проявляется в случае ЛБ27, нижний снимок). Заполнение индием (светлый контраст) пустот матрицы неполное, но, по нашим оценкам, составляет порядка 90-95\% - по крайней мере, на поверхности слоя. Учитывая метод заполнения матрицы индием с торцов из расплава, можно предположить аналогичное заполнение объема слоя СП-металлом.

Измерения сопротивления образцов $R$ проводились в режимах постоянного и переменного токов в магнитных полях $H \leq 10 \mathrm{kOe;} \mathrm{величина} \mathrm{измерительного}$ тока $I \leq 100 \mu \mathrm{A}$ выбиралась так, чтобы избежать его влияния на значения критических параметров СП перехода нанокомпозитов. Температурные и магнитополевые зависимости магнитного момента нанокомпозитов $M(T, H)$ изучались с использованием вибрационного магнитометра.

На рис. 3 показаны температурные зависимости сопротивления пленок ЛБ5 (1) и ЛБ27 (2); на вставке рис. 3 представлены те же зависимости в области сверхпроводящего перехода. Как видно из рис. 3, температурный ход сопротивления приготовленных образцов соответствует металлической зависимости при $T>20 \mathrm{~K}$, т. е. температурный коэффициент сопротивления не зависит от температуры, и при $T<20 \mathrm{~K}$ уменьшается. Логично предположить, что температурная зависимость сопротивления нанокомпозита определяется свойствами межгранульных мостиков, причем количество параллельных токоведущих путей оказывается много меньше числа последовательно включенных мостиков в силу тонкости пленочного образца. Каждый мостик представляет собой барьер на пути распространения тока, что связано либо с ограничением длины свободного пробега электронов, либо с уменьшением плотности электронных состояний 


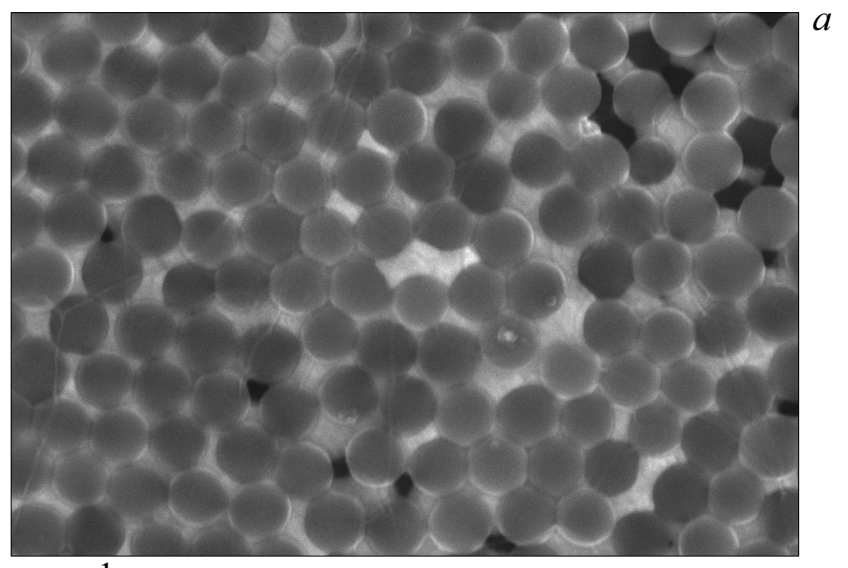

$1 \mu \mathrm{m}$

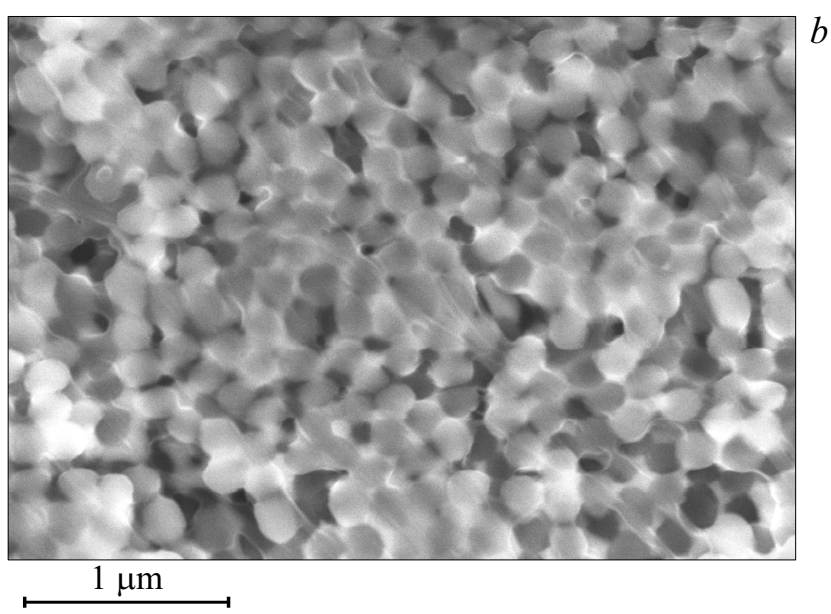

Рис. 2. SЕМ-фотографии пленок Ленгмюра-Блоджетт, заполненных индием. Вверху - пленка из 5 слоев, диаметр шаров $D=250 \mathrm{~nm}$; внизу — пленка из 27 слоев, $D=200 \mathrm{~nm}$. Заполнение In представляет светлый контраст.

в тонких мостиках, соединяющих гранулы индия [1]. Поэтому вся совокупность последовательно соединенных мостиков ответственна за значительное сопротивление образцов $R_{300 \mathrm{~K}} \approx 0.27 \mathrm{Ohm}$ (ЛБ5) и $0.1 \mathrm{Ohm}$ (ЛБ27). Разница в сопротивлении пленок закономерна и обусловлена большим количеством слоев и соответственно токопроводящих путей в ЛБ27.

При понижении температуры в сопротивлении образцов наблюдается переход исследуемых слоев в СП-состояние. Для ЛБ5 критическая температура СП-перехода, определенная по уровню $R=0.5 R_{N}$ $\left(R_{N}\right.$ - сопротивление образца непосредственно перед СП-переходом) $T_{\mathrm{c}}=3.5 \mathrm{~K}$. Ширина СП перехода в ЛБ5, определенная по разнице сопротивлений $\Delta R=0.9 R_{N}-0.1 R_{N}, \Delta T \approx 0.03 \mathrm{~K}$. Напротив, в ЛБ27 СП-переход сильно размыт, определенная аналогичным образом $\Delta T \approx 0.34 \mathrm{~K}$. На рис. 4 представлена зависимость $R(T)$ в образце ЛБ27 в области СП-перехода; на вставке рис. 4 показана температурная зависимость производной $d R / d T$. СП-переход имеет ярко выраженную ступенчатую структуру: можно выделить 2 пе- рехода с критическими температурами $T_{\mathrm{c} 1} \approx 3.57 \mathrm{~K}$ и $T_{\mathrm{c} 2} \approx 3.62 \mathrm{~K}$, что соответствует максимумам на зависимости $d R / d T(T)$. Неоднородный характер СП-пере-

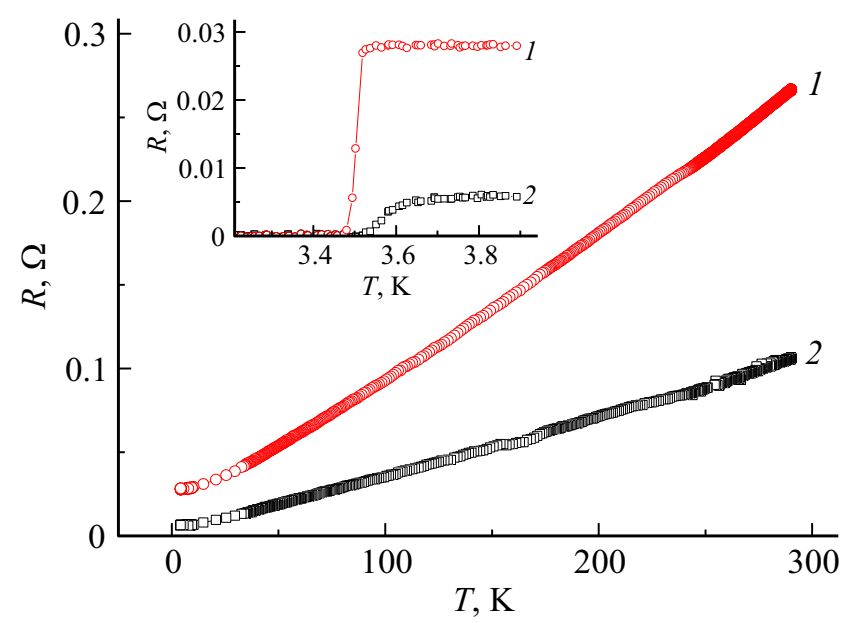

Рис. 3. Температурные зависимости сопротивления пленок LB5 (1) и LB27 (2) (5 и 27 слоев соответственно); на вставке - те же зависимости в области сверхпроводящего перехода.
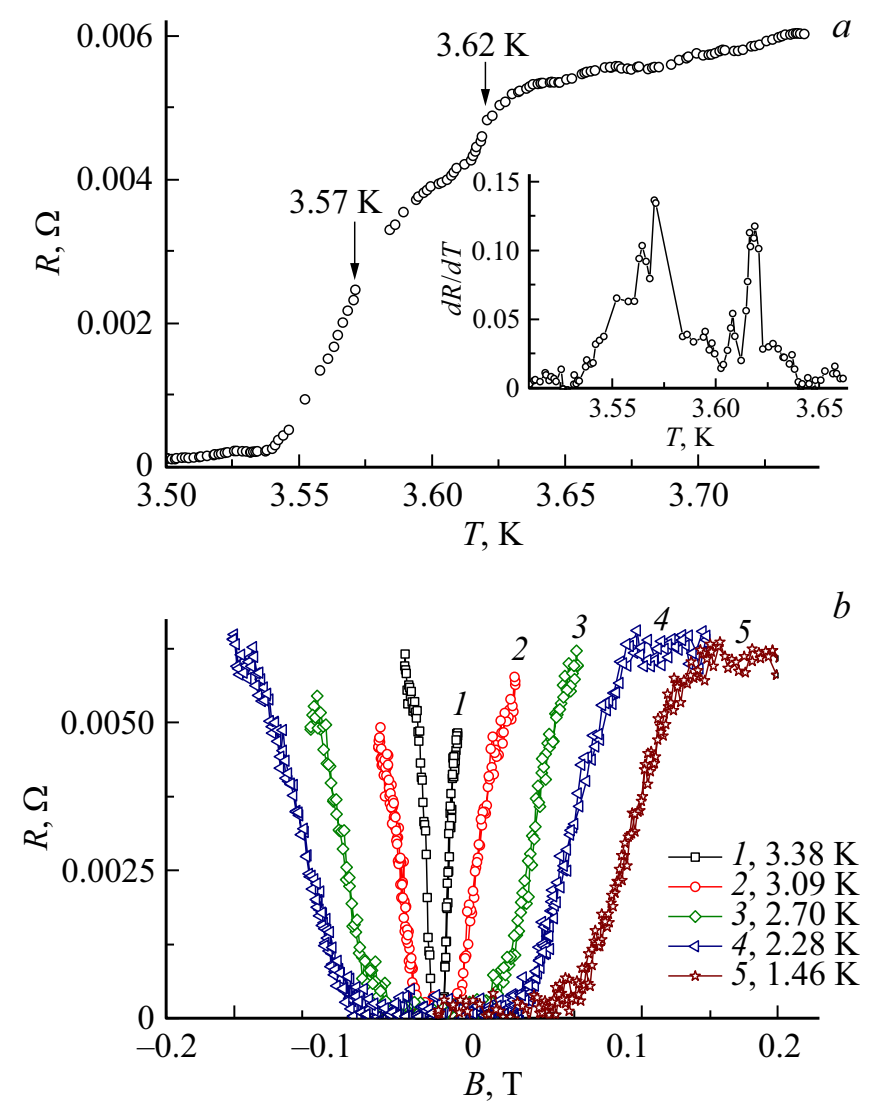

Рис. 4. $a-$ температурная зависимость сопротивления пленки ЛБ27 в области сверхпроводящего перехода; на вставке ее первая производная по температуре; $b-$ зависимость сопротивления пленки ЛБ27 от магнитного поля при различных температурах $T<T_{\mathrm{c}} . T=3.38 \mathrm{~K}(1), 3.09 \mathrm{~K}(2), 2.7 \mathrm{~K}$ (3), $2.28 \mathrm{~K}(4), 1.46 \mathrm{~K}(5)$. 


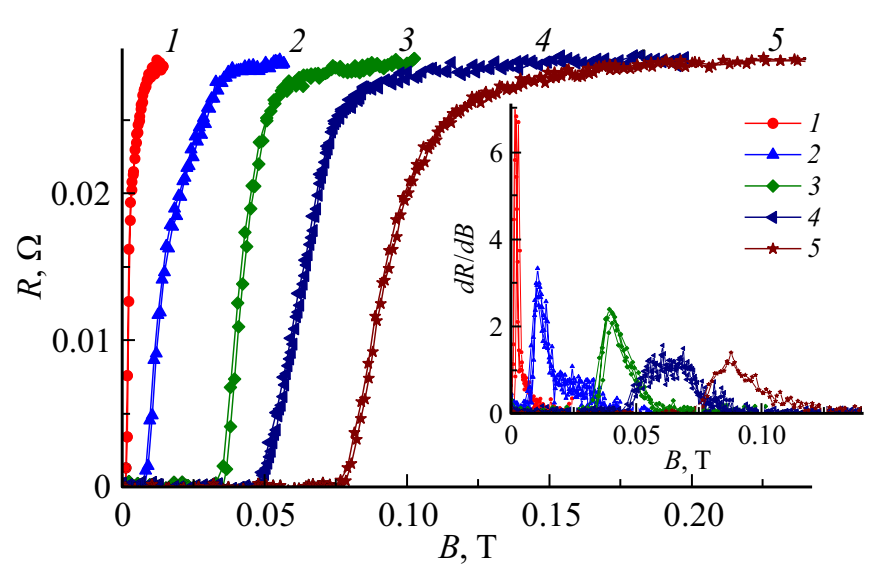

Рис. 5. Зависимость сопротивления пленки LB5 от магнитного поля при различных температурах $T<T_{\mathrm{c}}$. $T=3.38 \mathrm{~K}(1)$, $3.09 \mathrm{~K}(2), 2.7 \mathrm{~K}(3), 2.28 \mathrm{~K}(4), 1.46 \mathrm{~K}$ (5). На вставке: производная сопротивления пленки LB5 в зависимости от магнитного поля при тех же температурах.

хода связан, по-видимому, с различными по размеру составляющими токопроводящей сетки нанокомпозита. В этом случае, наблюдаемые $T_{c}$ можно сопоставить c характерными диаметрами токопроводящей индиевой сетки $d$. Используя экспериментальные данные [1], сопоставленные с теоретической размерной зависимостью $T_{c}(d)$ [4], получим выделенные минимальные диаметры In кластеров в нанокомпозите ЛБ27 $d_{1} \approx 30 \mathrm{~nm}$ и $d_{2} \approx 20 \mathrm{~nm}$. Отметим, что в зависимости $R(T)$ образца ЛБ 5 в области СП-перехода ступеней обнаружить не удалось, что, возможно, связано с малым уширением его СП перехода $\Delta T \approx 0.03 \mathrm{~K}$.

Как было показано в [1], низкотемпературным неоднородностям в зависимости $R(T)$ соответствуют особенности в зависимости $R(H)$ при $T<T_{\text {c. }}$ На рис. $4, b$ представлены зависимости сопротивления пленки ЛБ27 от магнитного поля при различных температурах $T<T_{\mathrm{c}}$. Как и следовало ожидать, магнитное поле $H>H_{\mathrm{c}}(T)$ возвращает сопротивление слоя в нормальное состояние. В то же время, тщательное изучение зависимостей $R(H)$ в образце ЛБ27 с помощью дифференцирования кривых и peak-аппроксимации не обнаружило на них особенностей, связанных с $T_{\mathrm{c} 1}$ и $T_{\mathrm{c} 2}$, наблюдаемых на зависимостях $R(T)$ для ЛБ27.

Рассмотрим рис. 5, на котором показаны экспериментальные зависимости сопротивления пленки LB5 от магнитного поля при различных температурах $T<T_{\mathrm{c}}$. На вставке показано, как производная сопротивления пленки LB5 зависит от магнитного поля. На некоторых кривых $R(T)$ в образце LB5 хорошо заметны изломы; анализ зависимостей с помощью дифференцирования показан на вставке к рис. 5. Практически на всех кривых $d R / d H$ можно выделить пики, соответствующие, по-видимому, характерным диаметрам, которые можно выделить в токопроводящей индиевой сетке нанокомпозита ЛБ5.
На рис. 6 представлены данные о зависимости критических магнитных полей в пленке ЛБ5 от температуры $H_{\mathrm{c}}(T)$ для 1 и 2 пиков в производной $d R / d B$ при различных температурах $T<T_{\mathrm{c}}$. Там же приведены значения $H_{\mathrm{c}}(T)$ для образца ЛБ27, определенные по уровню $R(H)=0.5 R_{N}$, где $R_{N}$ - сопротивление нанокомпозита в нормальном состоянии.

Качественно зависимости $H_{\mathrm{c}}(T)$, полученные из электрических $R(H, T)$ и магнитных $M(H, T)$ измерений, как и следовало ожидать, подобны. Количественные различия связаны с определением собственно $H_{\mathrm{c}}$ по уровню $R(H)=0.5 R_{N}$ (рис. 5), и $M(H)$ (рис. 7), где по величине $M(H) \approx 0$ определялось $H_{c}$, соответствующее полному вхождению магнитного потока в нанокомпозит. Эти различия могут быть связаны с тем, что при резистивных измерениях величина $T_{\mathrm{c}}$ определяется максимальным значением в массиве параллельных токопроводящих путей.

Сплошной кривой на рис. 6 показана аппроксимация данных $H_{\mathrm{c}}(T)$ для ЛБ27 по эмпирической формуле [5], при этом было использовано $T_{\mathrm{cm}} \approx 3.45 \mathrm{~K}$, как это следует из экспериментальной зависимости $H_{\mathrm{c}}(T)$ при $H_{\mathrm{c}}=0$

$$
H_{\mathrm{cm}}(T)=H_{\mathrm{c}} H(0)\left(1-\left(T / T_{\mathrm{cm}}\right)^{2} .\right.
$$

Определенное таким образом критическое магнитное поле при экстраполяции температуры к $T=0 \mathrm{~K}$ для ЛБ27 $H_{\mathrm{c}}(0) \approx 1.7 \mathrm{kOe}$. Аналогичная процедура для нанокомпозита ЛБ5 позволила получить значение $H_{\mathrm{c} 1}(0) \approx 1.11 \mathrm{kOe}$ и $H_{\mathrm{c} 2}(0) \approx 1.22 \mathrm{kOe} \quad\left(T_{\mathrm{cm}} \approx 3.4 \mathrm{~K}\right)$. Критическое магнитное поле исследованных образцов

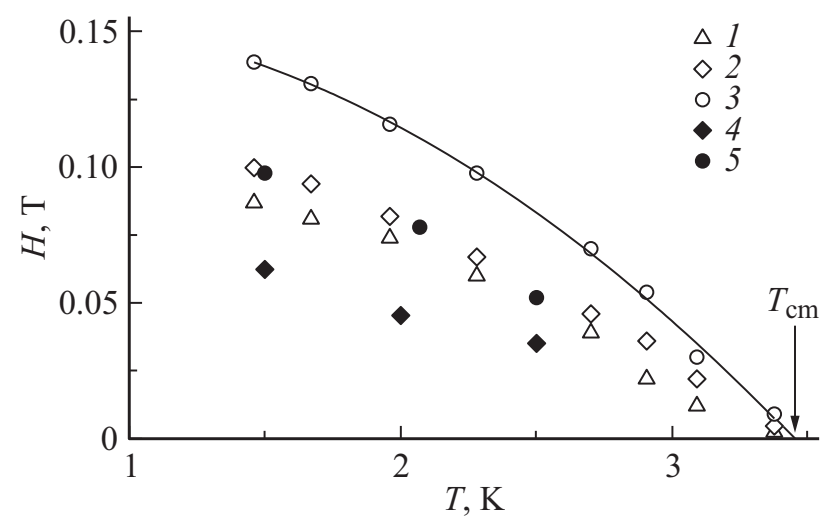

Рис. 6. Зависимость критических магнитных полей в пленке ЛБ5 от температуры $H_{\mathrm{c}}(T)$ для 1 и пиков (незаполненные ромбы (1) и треугольники (2) соответственно) в производной $d R / d B$ при различных температурах $T<T_{\mathrm{c}}$. Для ЛБ27 приведены значения $H_{\mathrm{c}}(T)$ (незаполненные кружки $\left.(3)\right)$, определенные по уровню $R(H)=0.5 R_{N}$, где $R_{N}-$ сопротивление слоя при переходе в нормальное состояние. Заполненные символы соответствуют критическим магнитным полям в пленке ЛБ5 (кружки, 4) и ЛБ27 (ромбы, 5), определенным из измерений магнитного момента при различных температурах $T<T_{\mathrm{c}}$. Сплошная кривая - аппроксимация данных $H_{\mathrm{c}}(T)$ для ЛБ27 по формуле $(1), T_{\mathrm{cm}}$ - критическая температура СП перехода, определенная из данных о магнитосопротивлении. 

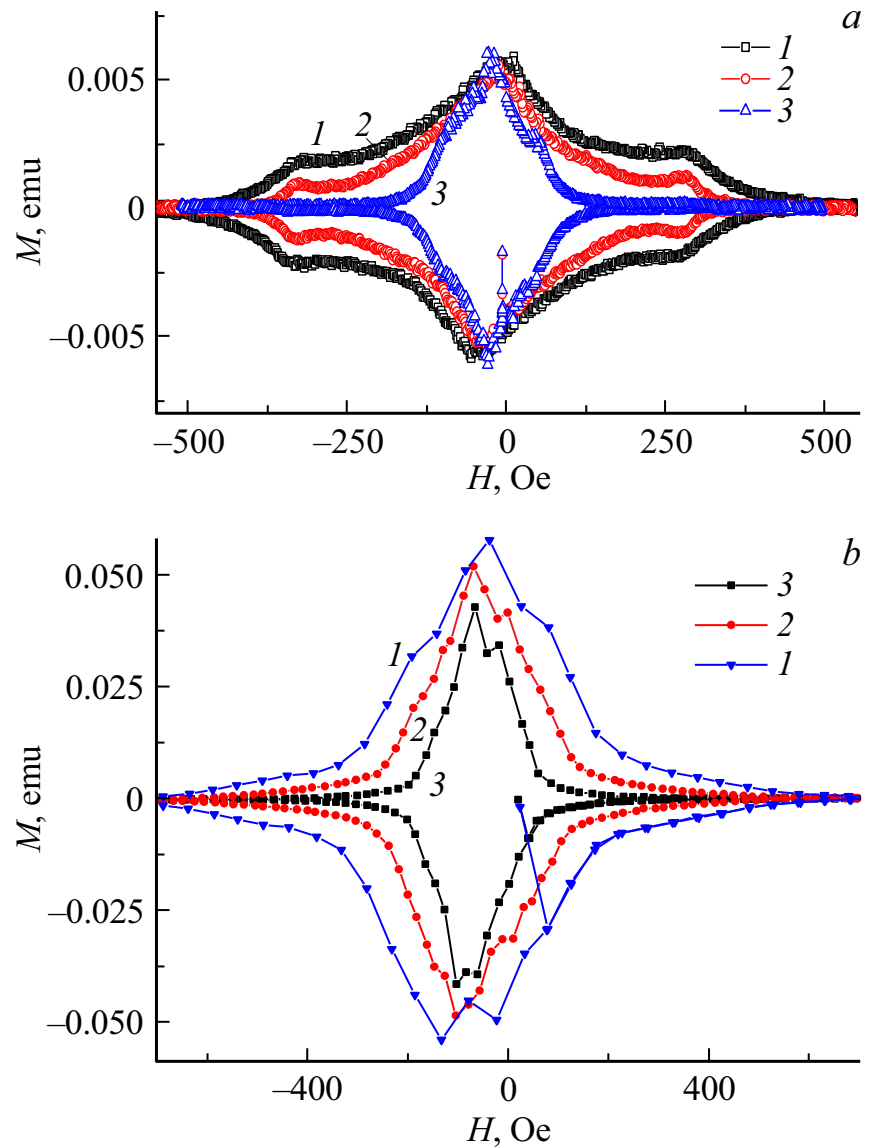

Рис. 7. $a-$ зависимость магнитного момента от магнитного поля для образца ЛБ5 при $T=1.5 \mathrm{~K}(1), 2.07 \mathrm{~K}(2)$ и $2.5 \mathrm{~K}(3)$; $b$ - зависимость магнитного момента от поля для образца ЛБ27 при $T=1.5 \mathrm{~K}(1), 2.07 \mathrm{~K}(2)$ и $2.5 \mathrm{~K}(3)$.

существенно превосходит значение $H_{\mathrm{c}}(0)=280$ Ое при $T=0 \mathrm{~K}$ для массивного индия, что говорит о формировании сверхпроводника II рода. Таким ообразом, размерный эффект в электропроводности указывает на присутствие значительной доли межгранульных мостиков, что также согласуется с формированием сверхпроводника II рода, характеризующегося большей величиной критического магнитного поля [1].

На рис. 7 представлены зависимости магнитного момента от магнитного поля для образцов ЛБ5 и ЛБ27 при различных температурах $T<T_{\mathrm{c}}$. В обоих случаях наблюдается ярко выраженный гистерезис, как и в случае массивного опала, заполненного индием [6], связанный с пиннингом магнитного потока в ячейках токопроводящей сети. Определенные из зависимостей $M(H)$ критические поля нанокомпозитов $H_{\mathrm{c}}$ показаны на рис. 6 . Максимум абсолютного значения намагниченности наблюдается вблизи нулевого магнитного поля, различия в абсолютной величине максимума для разных образцов объясняются различиями в форме и размерах изученных пленок.

При понижении температуры на зависимости $(M(H)$ в образце ЛБ5 все более отчетливо проявляется второй максимум (рис. 7,a). Можно допустить, что подобный пик наблюдается и в обр. ЛБ27, см. рис. 7, (b), однако для определенного утверждения необходимы измерения зависимости $M(H)$ при более низких температурах. Одно из возможных предположений о его природе, наряду с размерной зависимостью $H_{c}(d)$, связано с проявлением так называемого „пик-эффекта“, наблюдающегося в ряде классических (например, $\left.\mathrm{Nb}_{3} \mathrm{Sn}[7]\right)$ и высокотемпературных СП. Природа пик-эффекта, впрочем, до сих пор однозначно не установлена.

Обращает на себя внимание тот факт, что на зависимостях $M(H)$ при $T<T_{\mathrm{c}}$ отсутствуют квазипериодические скачки магнитного момента вплоть до $M \geq 0$, ранее обнаруженные в индиевых нанокомпозитах на основе массивного опала $[6,8]$. Возможно, это связано с меньшей упорядоченностью ЛБ-структур по сравнению с массивными опалами.

\section{3. Заключение}

В работе были впервые получены тонкие слои индиевых нанокомпозитов, изготовленных на основе тонких опалоподобных слоев, изучены их электрические и магнитные свойства, в том числе сверхпроводящие. Матрицы были изготовлены методом Ленгмюра-Блоджетт из сфер оксида кремния диаметром $d=200 \mathrm{~nm}$ и $d=250 \mathrm{~nm}$, In вводился в поры пленок из расплава под давлением, в результате чего создавалась трехмерная сетка наногранул индия. Измерения сопротивления и магнитного момента образцов в области сверхпроводящего перехода показали рост критических параметров наноструктурированного индия относительно массивного: получены значения критической температуры $T_{\mathrm{c}} \leq 3.62 \mathrm{~K}$ и критического магнитного поля, экстраполированного к $T=0 \mathrm{~K} H_{\mathrm{c}}(0) \leq 1.7$ кОе. В зависимостях сопротивления от температуры и от магнитного поля наблюдался ступенчатый переход в сверхпроводящее состояние, связанный со структурой нанокомпозита. Обнаружено, что на зависимостях магнитного момента исследованных образцов от магнитного поля при $T<T_{\mathrm{c}}$ наблюдается гистерезис, связанный с пиннингом магнитного потока в ячейках токопроводящей сети. При температурах $T \leq 2 \mathrm{~K}$ зависимость $M(H)$ в нанокомпозите ЛБ27 также имеет ступенчатый характер. Значения критических полей, полученные из электрических и магнитных измерений, удовлетворительно согласуются между собой.

Авторы выражают глубокую благодарность М. Бардосовой за изготовление матриц Лэнгмюра-Блоджетт.

\section{Список литературы}

[1] Д.В. Шамшур, А.В. Черняев, А.В. Фокин, С.Г. Романов. ФТТ 47, 11, 1927 (2005).

[2] G. Zhavnerko, G. Marletta. Mater. Sci. Eng. B 169, 1-3, 43 (2010). 
[3] В.Н. Богомолов, В.В. Журавлев, А.И. Задорожний, Е.В. Колла, Ю.И. Кумзеров. Письма в ЖЭТФ 36, 365 (1982).

[4] S. Matsuo, H. Sugiura, S. Noguchi. J. Low Temp. Phys. 15, 481 (1974).

[5] В.В. Шмидт. Введение в физику сверхпроводников. Наука, M. (1982).

[6] R.V. Parfeniev, D.V. Shamshur, A.V. Chernyaev, A.V. Fokin, S.G. Romanov. 9th Int. Symposium „Nanostructures: Physics and Technology“. St. Petersburg, Russia. (2001). P. 429-431.

[7] R. Lortz et al, Physical Rev. B 75, 094503 (2007).

[8] R.V. Parfeniev, D.V. Shamshur, M.S. Kononchuk, A.V. Chernyaev, S.G. Romanov, A.V. Fokin. 24 Int. Conf. Low Temp. Physics. Abstracts (2005). P. 235. Orlando, Florida, USA.

Редактор К.В. Емиев 